\title{
Coulomb Blockade and Kondo Effect in a Quantum Hall Antidot
}

\author{
H.-S. Sim,${ }^{1}$ M. Kataoka, ${ }^{2}$ Hangmo Yi, ${ }^{1}$ N. Y. Hwang, ${ }^{3}$ M.-S. Choi,${ }^{3}$ and S.-R. Eric Yang ${ }^{3}$ \\ ${ }^{1}$ School of Physics, Korea Institute for Advanced Study, \\ 207-43 Cheongryangri-dong, Dongdaemun-gu, Seoul 130-722, Korea \\ ${ }^{2}$ Cavendish Laboratory, Madingley Road, Cambridge CB3 OHE, United Kingdom and \\ ${ }^{3}$ Department of Physics, Korea University, 1 5-ka Anam-dong, Seoul 136-701, Korea
}

(Dated: November 1, 2018)

\begin{abstract}
We propose a general capacitive model for an antidot, which has two localized edge states with different spins in the quantum Hall regime. The capacitive coupling of localized excess charges, which are generated around the antidot due to magnetic flux quantization, and their effective spin fluctuation can result in Coulomb blockade, $h /(2 e)$ Aharonov-Bohm oscillations, and the Kondo effect. The resultant conductance is in qualitative agreement with recent experimental data.
\end{abstract}

PACS numbers: 73.23.Hk, 72.15.Qm, 73.43.-f

The Kondo effect arises due to many-body interactions between a localized spin and free electrons [1, 2]. Recently, there has been renewed interest in the effect as it was predicted [3, 4] and observed [5, 6, 7] in quantum dots. In a quantum dot, the localized spin is naturally provided when the dot has an odd number of electrons.

Quantum antidots in the integer quantum Hall regime have attracted recent interest in connection with experimental observations of the charging effect [8], $h /(2 e)$ Aharonov-Bohm (AB) oscillations [8, 9], and Kondo-like signatures [10]. In these systems, localized quantum Hall edge states are formed along an equipotential line of a "potential hill" which defines the antidot. As in quantum dots, electrostatic interaction of the localized antidot states may give rise to the charging effect. However, the magnetic flux quantization makes the antidots rather intriguing. When magnetic field $B$ changes adiabatically, each single-particle state encircling the antidot moves with respect to the antidot potential, adjusting the enclosed antidot area $S$ in order to keep the flux $B S$ constant [Fig. 1(b)]. This electron displacement results in charge imbalance around the antidot, i.e., local accumulation of excess charge $\delta q(B)$ [9], which is the source of the charging effect [8]. The accumulated $\delta q(B)$ is relaxed via single electron resonant tunnelings 11]. These tunneling events occur $\nu_{c}$ times within one $\mathrm{AB}$ period $\Delta B(=h / e S)$ when the antidot has $\nu_{c}$ localized edge states [12]. Also $\delta q(B)$ is periodic with the period $\Delta B$.

The origin of the Kondo-like signature in the antidots is not understood yet. One may naively consider that the spin-split single-particle antidot states support a localized spin. However, their $\mathrm{SU}(2)$ spin symmetry may be broken by the Zeeman energy and thus they can not cause the signature. Rather, many-body antidot states may play an important role.

In this Letter, we provide a theoretical model for the Kondo effect in a quantum Hall antidot system. As a natural way to incorporate the charging effect, capacitive interactions of excess charges with different spins are adopted, and the source-drain conductance $G(B)$ is computed within the model. We find that the effective spin flips of the excess charges can cause the Kondo effect. Within one $\mathrm{AB}$ period $\Delta B, G(B)$ can show approximately two normal resonances and one Kondo resonance, consistent with experimental data [10]. The two normal resonant tunneling events, involving spin-down electrons, are evenly spaced with varying $B$, constituting $h /(2 e) \mathrm{AB}$ oscillations, while the normal tunneling of spin-up electrons is Coulomb blockaded. These characteristic features of the resonances result from the interaction between the excess charges with different spins, and they can be tested by measuring conductance and higher cumulants of counting statistics such as shot noise.

Figure 11 shows the experimental setup and data in Ref. [10]. The data show a $2 e^{2} / h$ plateau $\left(\nu_{c} \sim 2\right)$ of $G(B)$ with a series of dips, which consist, within one $\mathrm{AB}$ period, of two normal $\mathrm{AB}$ resonance dips and one intermediate Kondo-like dip [see, eg., the data around $B=1.25 \mathrm{~T}$ in the inset of Fig. 1(c)]. These observations are consistent with the assumption that the antidot states come only from the two spin-split branches of the lowest Landau-level (LL) and that $\Delta B \ll B$. The system has both the localized antidot states and the extended edge states. The antidot hamiltonian can be written as $H_{\mathrm{AD}}=\sum_{m \sigma}\left(\hbar \omega_{c} / 2+E_{\sigma}^{Z}+V_{m}\right) c_{m \sigma}^{\dagger} c_{m \sigma}+$ $\sum_{m m^{\prime} n n^{\prime} \sigma \sigma^{\prime}} W_{m m^{\prime} n n^{\prime}} c_{n^{\prime} \sigma^{\prime}}^{\dagger} c_{n \sigma}^{\dagger} c_{m \sigma} c_{m^{\prime} \sigma^{\prime}}$, where $\omega_{c}$ is the cyclotron frequency, $E_{\sigma}^{Z}$ is the Zeeman energy, $V$ is the antidot potential energy (including a positive background term preserving total charge neutrality), $W$ is the Coulomb interaction, and $c_{m \sigma}^{\dagger}$ creates an electron with spin $\sigma$ in a localized single-particle state (enclosing $m$ magnetic flux quanta). The hamiltonian of the extended edge states is $H_{\text {edge }}=\sum_{i k \sigma} \epsilon_{i k \sigma} c_{i k \sigma}^{\dagger} c_{i k \sigma}$, where $c_{i k \sigma}^{\dagger}$ create an electron in an extended state (with momentum $k$, energy $\epsilon_{i k \sigma}$, spin $\sigma$, and index $i$ ). We only consider extended states coming from the first and the second LLs $\left(i \in\left\{M, M^{\prime}, N, N^{\prime}\right\}\right)$ [Fig. 1(a)]; the extended states of higher LLs have negligibly small tunneling amplitudes $V_{k m \sigma}^{i}$ to antidot. The total hamiltonian of the system is $H_{\text {tot }}=H_{\mathrm{AD}}+H_{\text {edge }}+H_{\text {tun }}$ with 

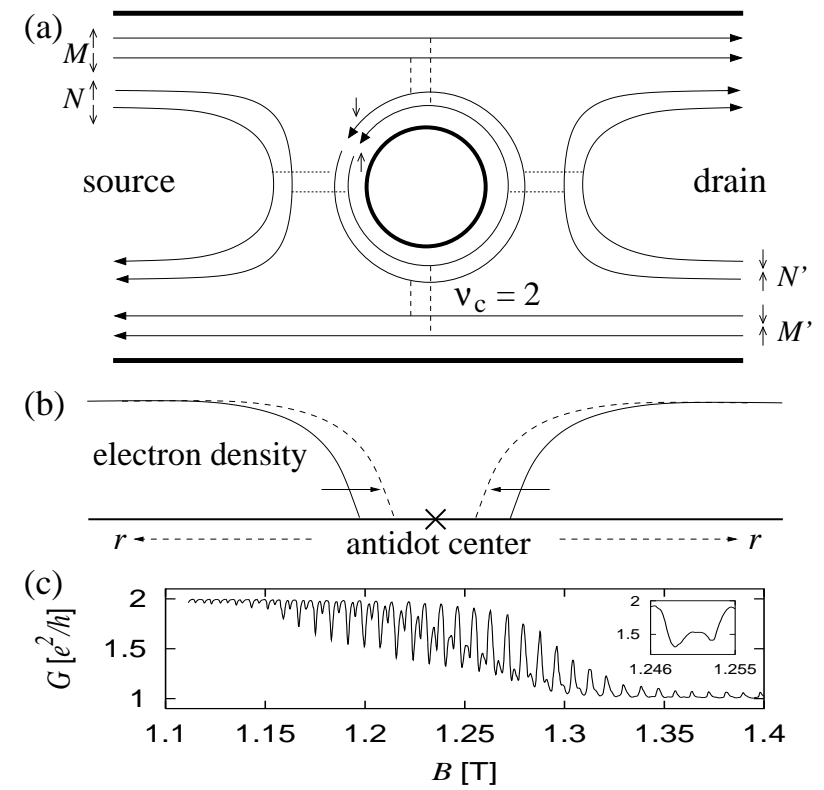

FIG. 1: (a) A quantum Hall antidot with antidot states and extended edge channels $\left(M, M^{\prime}, N, N^{\prime}\right)$ with their spins (solid arrows), which come from the 1st (the antidot states, $\left.M, M^{\prime}\right)$ and the 2nd Landau levels $\left(N, N^{\prime}\right)$. The intra and the inter-Landau level couplings are drawn as dashed and dotted lines, respectively. $B$ is applied perpendicularly to the plane. (b) Schematic diagram of electron density shift (solid arrows) around an antidot as $B$ increases; this generates local charge imbalance with respect to the background charge. (c) An experimental result under $25 \mathrm{mK}$ and zero bias (see Ref. [10]). Inset: $G(B)$ for one $\mathrm{AB}$ period around $B=1.25 \mathrm{~T}$.

\section{$H_{\text {tun }}=\sum_{i k m \sigma} V_{k m \sigma}^{i} c_{i k \sigma}^{\dagger} c_{m \sigma}+$ H.c.}

The energy $E_{\mathrm{AD}}$ of the antidot Hamiltonian $H_{\mathrm{AD}}$ varies with $B$ due to the formation [Fig. [1(b)] of localized excess charges $\delta q_{\sigma}(B)$. Since $\delta q_{\uparrow}$ and $\delta q_{\downarrow}$ are spatially separated from each other and from extended edge states by incompressible regions, one can approximate $E_{\mathrm{AD}}$ using an effective capacitance matrix $C$ as:

$$
E_{\mathrm{AD}}\left(\delta q_{\uparrow}(B), \delta q_{\downarrow}(B)\right)=\frac{1}{2} \sum_{\sigma \sigma^{\prime}} \delta q_{\sigma}\left(C^{-1}\right)_{\sigma \sigma^{\prime}} \delta q_{\sigma^{\prime}} .
$$

$\delta q_{\sigma}$ can have a form [13] of $\delta q_{\sigma}=-e N_{\sigma}^{\mathrm{AD}}-Q_{G \sigma}-$ $Q_{B \sigma}(B)$, where $N_{\sigma}^{\mathrm{AD}}$ is the total number of electrons with spin $\sigma$ occupying the antidot state, $Q_{G}$ is the "antidot-gate" charge (independent of $B$ ), and $Q_{B}(B)$ gives the dependence on $B . C_{\sigma \sigma^{\prime}}$ is a classical electrostatic quantity if alternating compressible and incompressible regions [14] are formed around the antidot. In other cases, it is a phenomenological parameter. We have $\left|C_{\uparrow \downarrow}\right|<C_{\uparrow \uparrow}<C_{\downarrow \downarrow}$, since $\delta q_{\downarrow}$ is located outer from antidot center than $\delta q_{\uparrow}$ due to its higher $E_{\sigma}^{Z} ;\left|C_{\uparrow \downarrow}\right|$ is the smallest as it is a mutual capacitance. Because $C_{\sigma \sigma^{\prime}}$ varies very slowly within one $\mathrm{AB}$ period, we will take it as constant. Note that $E_{\sigma}^{Z}$ can be counted in the definitions of $\delta q_{\sigma}$ and $C$, as it also causes the displacement and the sep-
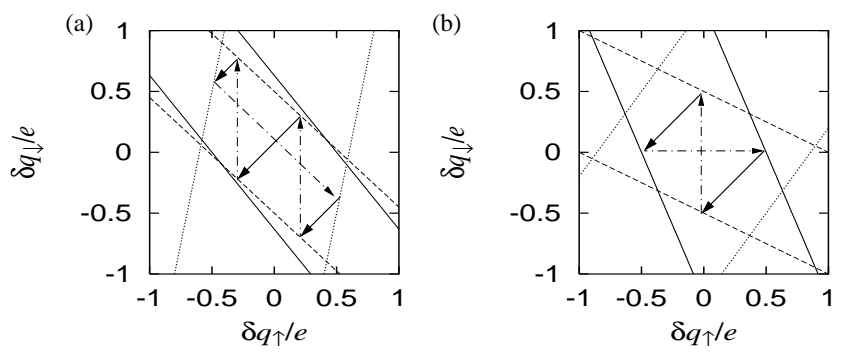

FIG. 2: $\left(\delta q_{\uparrow}, \delta q_{\downarrow}\right)$ evolves along a solid-arrow trajectory as $B$ increases. When one of resonance conditions is met, $\left(\delta q_{\uparrow}\right.$, $\delta q_{\downarrow}$ ) jumps following horizontal (normal spin-up electron tunneling), vertical (spin down), or diagonal dashed-dot arrows (Kondo resonance). Solid, dashed, and dotted lines indicate resonance conditions of Eqs. (2), (3), and (4), respectively. Two different parameters are used: (a) $\alpha \equiv\left|C_{\uparrow \downarrow}\right| / C_{\uparrow \uparrow}=0.95$ and (b) 0.5. For both the cases, we choose $C_{\downarrow \downarrow} / C_{\uparrow \uparrow}=1.2$.

aration of $\delta q_{\sigma}$ 's. Equation (10) is a good approximation for large-size antidots with $B \gg \Delta B$, and it is analogous to the constant interaction (CI) model of quantum dots [15. As in the CI model, the validity of Eq. (1) is tested within a Hartree-Fock approximation. Some terms independent of $N_{\sigma}^{\mathrm{AD}}$ have been ignored in Eq. (II) just like in the CI model. The control of $\delta q_{\sigma}$ by $B$ is reminiscent of the charge control by gate voltage in quantum dots.

Coulomb blockade prohibits tunneling unless one of the following conditions is satisfied. First, a normal resonance occurs whenever the antidot Hamiltonian is invariant under a single electron tunneling, i.e.,

$$
\begin{aligned}
& E_{\mathrm{AD}}\left(\delta q_{\uparrow} \pm e, \delta q_{\downarrow}\right)=E_{\mathrm{AD}}\left(\delta q_{\uparrow}, \delta q_{\downarrow}\right), \\
& E_{\mathrm{AD}}\left(\delta q_{\uparrow}, \delta q_{\downarrow} \pm e\right)=E_{\mathrm{AD}}\left(\delta q_{\uparrow}, \delta q_{\downarrow}\right) .
\end{aligned}
$$

Another allowed tunneling process is through a Kondo resonance, in which an electron tunnels into the antidot states and another electron with the opposite spin tunnels out via a virtual state. The condition for this is

$$
E_{\mathrm{AD}}\left(\delta q_{\uparrow} \pm e, \delta q_{\downarrow} \mp e\right)=E_{\mathrm{AD}}\left(\delta q_{\uparrow}, \delta q_{\downarrow}\right) .
$$

Among many available virtual states, the one with the lowest energy (thus with the biggest contribution) is either $\left(\delta q_{\uparrow} \pm e, \delta q_{\downarrow}\right)$ or $\left(\delta q_{\uparrow}, \delta q_{\downarrow} \mp e\right)$.

Assuming the antidot potential varies linearly within the scale of the magnetic length $l_{B}(\equiv \sqrt{h / e B}), \delta q_{\sigma}$ may be approximated as a linear function of $B$ between two adjacent tunneling (i.e., relaxation) events. Since the spatial separation of $\delta q_{\uparrow}$ and $\delta q_{\downarrow}$ is small compared with their average radius, $d \delta q_{\uparrow} / d B \simeq d \delta q_{\downarrow} / d B$. Therefore, as $B$ increases, the ground state value of $\left(\delta q_{\uparrow}, \delta q_{\downarrow}\right)$ evolves parallel to the line of $\delta q_{\uparrow}=\delta q_{\downarrow}$ until it jumps at one of the resonance conditions of Eqs. (2314) [see Fig. 2. The evolution trajectory of $\left(\delta q_{\uparrow}, \delta q_{\downarrow}\right)$ is periodic with pe$\operatorname{riod} \Delta B$. Depending on a parameter $\alpha \equiv\left|C_{\uparrow \downarrow}\right| / C_{\uparrow \uparrow}$ and 
initial values of $\left(\delta q_{\uparrow}, \delta q_{\downarrow}\right)$, two different types of trajectories are possible when $C_{\uparrow \uparrow}<C_{\downarrow \downarrow}$ : (i) two evenly spaced consecutive tunnelings of spin-down electrons as well as one intermediate Kondo resonance [Fig. 2(a)] or (ii) alternating tunnelings of spin-up and down electrons with an arbitrary phase difference [Fig. 2(b)]. If $\alpha=1$ (maximum), all trajectories are of type (i) regardless of the initial value of $\delta q_{\sigma}$. In the other extreme case $\alpha=0$ (minimum), i.e., $C_{\uparrow \downarrow}=0$, the two spin states are completely decoupled and all trajectories are of type (ii). For $0<\alpha<1$, both types are allowed depending on initial $\delta q_{\sigma}$. For larger $\alpha$, more trajectories follow the type (i). We remark that the experimental data [10] are consistent with the type (i): At high temperature, they show $h /(2 e) \mathrm{AB}$ oscillations, i.e., evenly spaced dips of $G(B)$ which appear two times per $\Delta B$. As the temperature is lowered, another dip appears approximately once every $\Delta B$. We argue that the experiment is in the parameter regime where type (i) trajectories are prevalent, i.e., $\alpha \sim 1$. The scale that separates the high and low temperature regimes is the Kondo temperature $T_{K}$.

We now describe in detail how the Kondo effect arises in our model. In the vicinity of a Kondo resonance condition, only the two lowest $\left[\left(\delta q_{\uparrow}, \delta q_{\downarrow}\right)\right.$ and $\left.\left(\delta q_{\uparrow}+e, \delta q_{\downarrow}-e\right)\right]$ and the next two excited states $\left[\left(\delta q_{\uparrow}+e, \delta q_{\downarrow}\right)\right.$ and $\left.\left(\delta q_{\uparrow}, \delta q_{\downarrow}-e\right)\right]$ of $H_{\mathrm{AD}}$ are important; we ignore all the other excited states, which may affect the Kondo effect only slightly as in multilevel quantum dots 16. Using these four states one can map $H_{\mathrm{AD}}$ into the Anderson impurity model, given by a truncated hamiltonian $H_{\mathrm{imp}}=\sum_{\sigma} \epsilon_{\sigma} d_{\sigma}^{\dagger} d_{\sigma}+U d_{\uparrow}^{\dagger} d_{\downarrow}^{\dagger} d_{\downarrow} d_{\uparrow}$, where $d_{\sigma}^{\dagger}$ creates an electron in the impurity site. The two lowest states constitute the two singly occupied impurity states, while the next two excited states do the empty and doubly occupied states. Defining $E_{\text {empty }} \equiv E_{\mathrm{AD}}\left(\delta q_{\uparrow}+e, \delta q_{\downarrow}\right)$, we get $\epsilon_{\uparrow}=E_{\mathrm{AD}}\left(\delta q_{\uparrow}, \delta q_{\downarrow}\right)-E_{\mathrm{empty}}, \epsilon_{\downarrow}=E_{\mathrm{AD}}\left(\delta q_{\uparrow}+e, \delta q_{\downarrow}-e\right)-$ $E_{\text {empty }}$, and $U+\epsilon_{\uparrow}+\epsilon_{\downarrow}=E_{\mathrm{AD}}\left(\delta q_{\uparrow}, \delta q_{\downarrow}-e\right)-E_{\text {empty }}$. Here, $\Delta \epsilon \equiv \epsilon_{\uparrow}-\epsilon_{\downarrow}$ behaves as an effective Zeeman energy of the impurity site; $\Delta \epsilon=0$ at the Kondo resonance condition. $H_{\text {tot }}$ can now be mapped into the impurity coupled to the extended edge states by amplitude $V_{k \sigma}^{i}$ :

$$
H_{\mathrm{K}}=H_{\text {imp }}+H_{\text {edge }}+\sum_{i k \sigma} V_{k \sigma}^{i} c_{i k \sigma}^{\dagger} d_{\sigma}+\text { H.c. }
$$

One can estimate energy scales from experimental data. Since only down-spin electrons cause the normal resonances in the type (i) trajectories, we rewrite Eq. (11) as $E_{\mathrm{AD}}\left(\delta q_{\uparrow}, \delta q_{\downarrow}\right)=\left(\delta q_{\downarrow}+\alpha \delta q_{\uparrow}\right)^{2} /\left(2 C_{\text {out }}\right)+\delta q_{\uparrow}^{2} /\left(2 C_{\text {in }}\right)$, where $C_{\text {out }}=C_{\downarrow \downarrow}-\alpha\left|C_{\uparrow \downarrow}\right|$ and $C_{\text {in }}=C_{\uparrow \uparrow}$ [17]. One can then easily see that $e^{2} / C_{\text {out }}$ corresponds to the charging energy $(\sim 60 \mu \mathrm{eV})$ measured in Ref. [10]. Note that $-\epsilon_{\sigma}, U+\epsilon_{\sigma} \sim e^{2} /\left(2 C_{\text {out }}\right)$. When the effective Zeeman energy $\Delta \epsilon$ has a finite value, the Kondo resonance is split and suppressed [18, 19], and thus the Kondo signature of $G(B)$ can only appear within a certain range $\delta B$ in one $A B$ period $\Delta B$. For $\alpha=1$, the Kondo res-

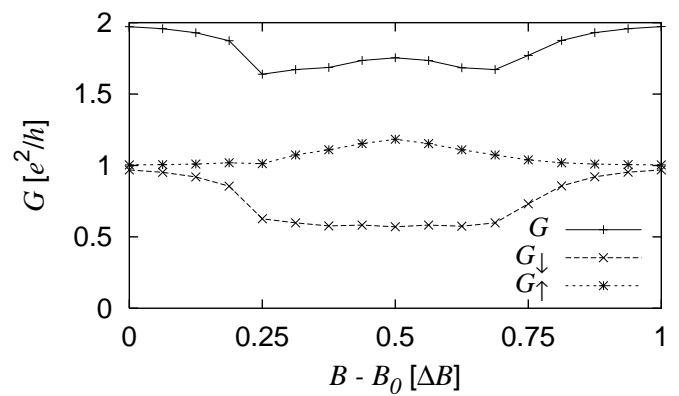

FIG. 3: Calculated $G(B)$ and $G_{\sigma}(B)$ around a magnetic field $B_{0}(\sim 1 \mathrm{~T}) . G(B)$ matches well the experimental data, eg., the inset in Fig. 1(c).

onance occurs at $\delta q_{\uparrow}= \pm e / 2$, and in its vicinity where $\delta q_{\uparrow}= \pm(1 / 2-p) e$, we get $\Delta \epsilon=p e^{2} / C_{\text {in }}$. From this, one can roughly estimate that $e^{2} / C_{\text {in }}$ is of the order of 10 $\mu e V$ in the experimental situation [10], where $\Delta \epsilon \lesssim 10$ $\mu \mathrm{eV}$ (the energy scale of zero bias anomaly), the width $\delta B$ of the Kondo dip $\simeq \Delta B / 4$, and $p \simeq \delta B / \Delta B$.

The resonance width $\Gamma_{\sigma}(E) \equiv \sum_{i} \Gamma_{\sigma}^{i}(E)$ has a spin dependence since $V_{k \uparrow}^{i=M\left(M^{\prime}\right)} \neq V_{k \downarrow}^{i=M\left(M^{\prime}\right)}$ (Fig. 1), where $\Gamma_{\sigma}^{i}(E)=2 \pi \sum_{k}\left|V_{k \sigma}^{i}\right|^{2} \delta\left(E-\epsilon_{i k \sigma}\right)$. We will ignore $k$ dependence of $V_{k \sigma}^{i}$ for simplicity. The spin-dependent $\Gamma_{\sigma}$ 's renormalize the effective Zeeman energy as $\Delta \tilde{\epsilon}\left(\delta q_{\uparrow}, \delta q_{\downarrow}\right)$, as in the quantum dots coupled to ferromagnetic leads [20]. This changes the Kondo resonance condition to

$$
E_{\mathrm{AD}}\left(\delta q_{\uparrow} \pm e, \delta q_{\downarrow} \mp e\right)=E_{\mathrm{AD}}\left(\delta q_{\uparrow}, \delta q_{\downarrow}\right)+\Delta \tilde{\epsilon},
$$

instead of Eq. (4). When Eq. (6) is satisfied, there is no renormalized effective Zeeman splitting so that one can estimate 21] $T_{K} \sim(\sqrt{\Gamma U} / 2) \exp (\pi \epsilon(U+\epsilon) / \Gamma U)$, where $\Gamma=\left(\Gamma_{\uparrow}+\Gamma_{\lrcorner}\right) / 2$. By using $\alpha=1$ and estimating energy scales 22], we perform the numerical renormalization group (NRG) calculation 2, 23] and find $T_{K} \sim 1$ $\mu \mathrm{eV}$, which has the same order of magnitude with the zero-bias anomaly of the Kondo-like data 10].

Now we derive the conductance at zero temperature and zero bias [4, 24] as $G(B)=\sum_{\sigma} G_{\sigma}(B)$ where $G_{\sigma}=\left(e^{2} / h\right)\left(1+T_{\sigma} \sin ^{2} \theta_{\sigma}\right)$. Here, $T_{\sigma} \equiv 4\left(\Gamma_{\sigma}^{i=N} \Gamma_{\sigma}^{i=N^{\prime}}-\right.$ $\left.\Gamma_{\sigma}^{i=M} \Gamma_{\sigma}^{i=M^{\prime}}\right) / \Gamma_{\sigma}^{2}$ and $\theta_{\sigma} \equiv \operatorname{Im} \ln \mathcal{G}_{\sigma}(E+i \delta)$, where $\mathcal{G}_{\sigma}(E)$ is the Green's function of the impurity site. $\Gamma_{\sigma}^{i}$ 's can be estimated 22 from the experiments in the following way: Since $G(B)$ shows dips below $2 e^{2} / h$ [Fig. 1(c)] and the excess charges follow a type (i) trajectory, we expect $\Gamma_{\downarrow}^{i=M, M^{\prime}}>\Gamma_{\downarrow}^{i=N, N^{\prime}}$ and $T_{\downarrow}<0$, i.e., the down spin electrons favor the backward scattering. On the other hand, the $\Delta B$ periodic peaks at stronger magnetic fields near the plateau of $G \simeq e^{2} / h$ may be interpreted as normal resonances of spin-up electrons, and $\Gamma_{\sigma}^{i=M, M^{\prime}}$ becomes smaller as $B$ decreases. One thus obtains that $\Gamma_{\uparrow}^{i=M, M^{\prime}}<\Gamma_{\uparrow}^{i=N, N^{\prime}}$ and $T_{\uparrow}>0$ for weaker $B$ where $G>e^{2} / h$. Then we can calculate $G(B)$ for an AB period 
by using the NRG results of $\sin ^{2} \theta_{\sigma}$ obtained for $\alpha=1$. The result (see Fig. 3) is in qualitatively good agreement with the experimental data, eg., the inset of Fig. 1(c).

Figure 3 shows a spin-dependent behavior of $G_{\sigma}(B)$. $G_{\uparrow}$ has a Kondo peak, while $G_{\downarrow}$ shows a mixture of two normal and one intermediate Kondo resonance dips. As a result, at the center of the Kondo resonance, even if the unitary Kondo limit is reached, $G$ can have a larger value than $e^{2} / h$. The center and the width of the Kondo resonance in $G_{\sigma}(B)$ is governed by Eq. (6) and $e^{2} / C_{\mathrm{in}}$, respectively. This spin-dependent behavior results from the type (i) trajectory and multiple extended edge states (i.e., the sign of $T_{\sigma}$ ), and is an interesting feature of the antidot Kondo effect. Our predictions on $G_{\sigma}(B)$ may be tested by measuring conductance and higher cumulants of counting statistics such as shot noise [25], which can resolve $G$ into $G_{\uparrow}$ and $G_{\downarrow}$.

In the broad range of many $\mathrm{AB}$ periods, as $B$ increases, the spin-down antidot state becomes more strongly coupled to the extended states with $i=M, M^{\prime}$ and then eventually disappears. Then, $G(B)$ decreases from $2 e^{2} / h$ to $e^{2} / h$, and the Kondo effect may become enhanced at first and then suppressed around $B$ where $G \simeq e^{2} / h$. Note that our model can also naturally explain $h /(2 e)$ $\mathrm{AB}$ oscillations without any Kondo signature at higher $B \sim 3 \mathrm{~T}[9]$ where the spin-up antidot state is decoupled with all extended edge states.

Finally, we note that as a Kondo resonance is crossed by increasing $B$, the total electron spin of the ground state decreases [see Fig. 2(a)]. To understand the microscopic origin of this behavior, we perform a Hartree-Fock (HF) calculation [26], in which the electron antidot system is transformed into a system confining holes by the mapping $c_{m \sigma} \rightarrow h_{m \sigma}^{\dagger}$ and $c_{m \sigma}^{\dagger} \rightarrow h_{m \sigma}$. Here, $h_{m \sigma}^{\dagger}$ creates a hole. We use an inverse bell-shape confinement potential such as $a r^{2}$ for $r<r_{c}$ and $b+c / r^{2}$ for $r>r_{c}$, so that hole states near the edge are affected by the $1 / r^{2}$ potential. Our HF calculation with about 50 confined holes shows that there appear the ground state transitions from $\left|N_{\downarrow}, N_{\uparrow}\right\rangle$ to $\left|N_{\downarrow}-1, N_{\uparrow}+1\right\rangle$ with increasing $B$ when the curvature of the confinement potential near the edge of the system is negative. $\left|N_{\downarrow}, N_{\uparrow}\right\rangle$ is a maximum density droplet state [26] with $N_{\uparrow}$ spin-up and $N_{\downarrow}$ spindown holes $\left(N_{\downarrow}>N_{\uparrow}\right.$ due to the Zeeman energy) and the droplet size is $\sim \sqrt{2 N_{\downarrow}} l_{B}$. The transitions, where the total electron spin decreases, occur because for the negative-curvature potential, the total confinement energy of holes increases faster than the total Coulomb energy as $B$ increases. This causes the hole droplet to minimize its size. Although the HF calculation with a relatively small number of holes can not be directly compared with large-size antidots, it demonstrates the existence of the spin-flip ground state transition.

To conclude, we have constructed a capacitive model which can describe many-body antidot states in integer quantum Hall regimes, and predicted Coulomb blockade and characteristic AB oscillations with the Kondo effect. Our model may be applicable to large-size quantum dots or rings in $\nu=2$ quantum Hall regimes [27] as well.

HSS and MK thank C. J. B. Ford and H. Schomerus for helpful discussions and their hospitality in Cambridge and Dresden. The discussions with K. Kikoin, K. Kang, and T. S. Kim are gratefully acknowledged. We were supported by SKORE-A (HY and MSC), eSSC (MSC), and QSRC at Dongguk University (SREY).

[1] J. Kondo, Prog. Theor. Phys. 32, 37 (1964).

[2] A. C. Hewson, The Kondo Problem to Heavy Fermions (Cambridge University Press, Cambridge, 1993).

[3] L. I. Glazman and M. E. Raikh, JETP Lett. 47, 452 (1988).

[4] T. K. Ng and P. A. Lee, Phys. Rev. Lett. 61, 1768 (1988).

[5] D. Goldhaber-Gordon et al., Nature 391, 156 (1998).

[6] S. M. Cronenwett, T. H. Oosterkamp, and L. P. Kouwenhoven, Science 281, 540 (1998).

[7] W. G. van der Wiel et al., Science 289, 2105 (2000).

[8] C. J. B. Ford et al., Phys. Rev. B 49, 17456 (1994); A. S. Sachrajda et al., Phys. Rev. B 50, 10856 (1994); M. Kataoka et al., Phys. Rev. Lett. 83, 160 (1999).

[9] M. Kataoka et al., Phys. Rev. B 62, R4817 (2000).

[10] M. Kataoka et al., Phys. Rev. Lett. 89, 226803 (2002).

[11] In fractional quantum Hall regimes, fractional charge relaxations may be allowed [see D. J. Thouless and Y. Gefen, Phys. Rev. Lett. 66, 806 (1991)].

[12] AB oscillations of tunneling conductance were investigated in a quantum Hall line junction with $\nu_{c}=1$ [see E.-A. Kim and E. Fradkin, cond-mat/0305693.

[13] If only incompressible regions (or maximum-density droplets of holes) are formed around the antidot, one estimates $\delta q_{\sigma} \simeq\left(e n_{e}\right) \delta S$ in terms of electron density $n_{e}=$ $e B / h$ per spin and area shift $\delta S$ caused by field change $\delta B \equiv B-B_{0}(<\Delta B)$ from a given $B_{0}$. Using $\delta(B S)=0$, one finds $Q_{B \sigma}(B)=Q_{B \sigma}\left(B_{0}\right)-e\left(B-B_{0}\right) / \Delta B$. This estimation can be modified due to charge redistribution, if compressible regions are also formed.

[14] D. B. Chklovskii, B. I. Shklovskii, and L. I. Glazman, Phys. Rev. B 46, 4026 (1992).

[15] For a recent review, see L. I. Glazman and M. Pustilnik, cond-mat/0302159

[16] T. Inoshita et al., Phys. Rev. B 48, 14725 (1993); A. Levy Yeyati, F. Flores, and A. Martín-Rodero, Phys. Rev. Lett. 83, 600 (1999); D. Boese, W. Hofstetter, and H. Schoeller, Phys. Rev. B 66, 125315 (2002).

[17] When the antidot states are the alternating compressible and incompressible regions, $\delta q_{\uparrow}$ in the inner compressible region $(\mathrm{CR})$ can be screened by the outer $\mathrm{CR}$ with a screening factor $\eta$ (for perfect screening, $\eta=1$ ). Then charges of $\delta q_{\uparrow}$ and $\delta q_{\downarrow}+\eta \delta q_{\uparrow}$ are accumulated at the inner and outer CRs, respectively [9]. They can be described by the rewritten $E_{\mathrm{AD}}$ with $C_{\text {out }}, C_{\text {in }}$, and $\alpha \equiv \eta$.

[18] Y. Meir, N. S. Wingreen, and P. A. Lee, Phys. Rev. Lett. 70, 2601 (1993).

[19] In Ref. [10], the resolution is not good enough to see the splitting of Kondo signatures at a finite bias.

[20] P. Zhang et al., Phys. Rev. Lett. 89, 286803 (2002); 
R. López and D. Sánchez, Phys. Rev. Lett. 90, 116602 (2003); J. Martinek et al., cond-mat/0210006

[21] F. D. M. Haldane, Phys. Rev. Lett. 40, 416 (1978).

[22] We estimate $e^{2} / C_{\text {out }}=60 \mu \mathrm{eV}, e^{2} / C_{\text {in }}=12 \mu \mathrm{eV}$, and $\Gamma_{\downarrow}=22 \mu \mathrm{eV}$ (full width of half maximum) from the experimental data [10]. To obtain the ratios of $\Gamma_{\sigma}^{i}$ 's $\left[\propto \exp \left(-2 l_{i \sigma} / l_{B}\right)\right]$, we also crudely estimate hopping distances $l_{i \sigma}$ of the antidot state with spin $\sigma$ to extended edge channels with index $i$ as $l_{M \downarrow}=l_{B}, l_{M \uparrow}=1.7 l_{B}$, $l_{N \uparrow}=l_{N \downarrow}=1.5 l_{B}$, and $l_{M^{\prime}\left(N^{\prime}\right) \sigma}=l_{M(N) \sigma}$. Note that $l_{B} \sim 25 \mathrm{~nm}$ when $B \sim 1 \mathrm{~T}$.

[23] K. G. Wilson, Rev. Mod. Phys. 47, 773 (1975); T. A. Costi et al., J. Phys.: Cond. Mat. 6, 2519 (1994).
[24] G. Kirczenow, Phys. Rev. B 50, 1649 (1994).

[25] Ya. M. Blanter and and M. Büttiker, Phys. Rep. 336, 1 (2000).

[26] S.-R. E. Yang, A. H. MacDonald, and M. D. Johnson, Phys. Rev. Lett. 71, 3194 (1993); A. H. MacDonald, S.R. E. Yang, and M. D. Johnson, Aust. J. Phys. 46, 345 (1993).

[27] A. A. M. Staring et al., Phys. Rev. B 46, 12869 (1992); T. Heinzel et al., Phys. Rev. B 50, 15113 (1994); J. P. Bird et al., Phys. Rev. B 53, 3642 (1996); M. Keller et al., Phys. Rev. B 64, 033302 (2001). 